\title{
The effect of the system polydispersity on voltammograms of nanoparticles electrooxidation
}

\author{
Kh. Z. Brainina • L. G. Galperin • E. V. Vikulova • A. L. Galperin
}

Received: 26 July 2012 /Revised: 14 August 2012 / Accepted: 17 August 2012 /Published online: 4 September 2012

(C) Springer-Verlag 2012

\begin{abstract}
The paper proposes a mathematical model describing electrooxidation of a polydisperse system of metal nanoparticles from the surface of an indifferent macroelectrode. It is shown that the degree of dispersion of a nanoparticle ensemble affects the shape of oxidation voltammograms. When the degree of dispersion rises and the average size of nanoparticles becomes smaller, the range of oxidation potentials increases. The results of the experimental study of electrooxidation of gold nanoparticles with different degrees of dispersion are given. The particles were localized on the surface of graphite screen-printed electrodes. A good agreement between the parameters of the experimental and calculated voltammograms confirms the correctness of the proposed model.
\end{abstract}

Keywords Nanoparticles · Polydisperse systems ·

Electrooxidation

Electronic supplementary material The online version of this article (doi:10.1007/s10008-012-1852-y) contains supplementary material, which is available to authorized users.

K. Z. Brainina $(\bowtie) \cdot$ E. V. Vikulova

Ural State University of Economics,

8 Marta St, 62,

Ekaterinburg 620144, Russia

e-mail: baz@usue.ru

K. Z. Brainina • L. G. Galperin

Ural Federal University,

Mira St 19, A-203,

Ekaterinburg 620002, Russia

L. G. Galperin

e-mail: lhalp@k66.ru

A. L. Galperin

Ural Federal University,

Lenin Avenue 51,

Ekaterinburg 620083, Russia

\section{Introduction}

The development of nanotechnology and the expansion of practical uses for nanomaterials require comprehensive information about nanoparticle properties, which may differ significantly from the properties of macroparticles. Investigation of the properties of metal nanomaterials by electrochemical methods is proposed by comparing the results of mathematical modeling with experimental voltammetric curves obtained by dissolving metal nanoparticles from the surface of an indifferent electrode. In the majority of the proposed models for these processes, the focus is on the effect of the geometry of monodisperse deposit on the electrode process [1, 2]. In [3-6] when studying the kinetics of the electrode process, the measured current is identified with the performance of "a plane source," - an electrode, to avoid detailing the location of particles on its surface. However, the subject of consideration still remains monodisperse systems. Meanwhile, in real terms, both sols and electrode surface contain a mix of particles that is polydisperse. Considering that the properties of nanoparticles of varying sizes differ (in fact, the smaller the size, the more significant the difference), it is important to take into account not only the average size of nanoparticles but also the distribution of particles according to size. For example, two sols with the same average size but different degrees of dispersion will exhibit different toxicity in biological structures due to the presence of small particles in polydisperse sol that are able to penetrate cell membranes quite easily [7]. Therefore, in general, degree of dispersity should be taken into account interpreting the processes involving polydisperse system participation. This assumption is supported by observed differences in the shape of voltammograms calculated with the use of a mono-model and obtained experimentally $[4-6,8]$.

The goals of the proposed work paper are to accomplish the following: mathematical modeling of the electrooxidation process of polydisperse nanosystems, a comparison of 
calculated and experimental data by studying electrooxidation of nanoparticle ensembles with different degrees of dispersion, and a demonstration of possible uses of electrochemical data to evaluate the degree of dispersion of nanoparticle ensembles and the average particle radius.

\section{Theory calculation}

Electrooxidation of polydisperse systems can be described by using the kinetic equation for the distribution density of particles by radius $[9,10]$.

We assume that at a given time, the electrode surface contains a polydisperse mix of spherical nanoparticles with the radii ranging from

$r_{\min } \leq r \leq r_{\max }$

with density normalized per unit $f_{0}(r)$

$\int_{0}^{\infty} f_{0}(r) d r=1$

The range of integration by $r$ is defined on $(0, \infty)$. It can always be done assuming that $f_{0}(r)=0$ if $r_{\min }<r$ and $r>r_{\max }$ [9].
The particle size distribution in polydisperse mixes is usually described by normal law. During electrodissolution of particle distribution, function $f(r, t)$ evolves in accordance with the kinetic equation $[9,10]$ :

$\frac{\partial f}{\partial t}+\frac{\partial}{\partial r}\{W(r, t) f\}=0$,

with the initial condition

$\left.f(r, t)\right|_{t=0}=f_{0}(r)$.

Unlike $f_{0}(r)$, function $f(r, t)$ is not normalized per unit. Its integral over the entire range of values, and $r$ determines the quantity of particles that have not dissolved by the time $t[9,10]$ :

$N=N_{0} \int_{0}^{\infty} f(r, t) \mathrm{d} r$

In order to calculate the change in the radius of a single particle $W(r, t)$, the equation of charge balance is used

$4 \pi r^{2} \frac{\mathrm{d} r}{\mathrm{~d} t} \frac{\rho F}{M}=4 \pi r^{2} W \frac{\rho F}{M}=i_{\mathrm{p}}$

According to [3], an equation for calculating current value $i_{\mathrm{p}}$ proportional to the particle surface area is

$i_{\mathrm{p}}=\mathrm{nFk}_{S} 4 \pi r^{2}\left\{a_{\mathrm{M}} \mathrm{e}\left[\frac{n \beta F}{\mathrm{RT}}\left(E-E_{0}\right)+\frac{\beta \Delta G^{\circ}}{\mathrm{RT}}\right]-c(t) \mathrm{e}\left[-\frac{n \alpha F}{\mathrm{RT}}\left(E-E_{0}\right)-\frac{\alpha \Delta G^{\circ}}{\mathrm{RT}}\right]\right\}$

where $E=E(t)=E_{\mathrm{in}}+v \mathrm{t}$ is current potential, $r$ is the particle radius in the monodisperse system.

Equations 5 and 6 are involved in an equation for calculating the particle dissolution rate

$W(t)=\frac{M}{4 \pi r^{2} \rho F} i_{\mathrm{p}}=A \varphi(t)$,

$A=\mathrm{const}=\frac{\mathrm{nMk}_{s}}{\rho}$,
$\varphi(t)=\left\{a_{M} e\left[\frac{n \beta F}{\mathrm{RT}}\left(E-E_{0}\right)+\frac{\beta \Delta G^{\circ}}{\mathrm{RT}}\right]-c(t) \mathrm{e}\left[-\frac{\mathrm{n} \alpha \mathrm{F}}{\mathrm{RT}}\left(E-E_{0}\right)-\frac{\alpha \Delta G^{\circ}}{\mathrm{RT}}\right]\right\}$

Since the particle dissolution rate (7) depends only on time, Eq. 3 can be solved with the Laplace transform by variable $r, f(r, t) \Rightarrow \bar{f}(p, t)$ [9]. Thus, Eq. 4 comes as

$\frac{\mathrm{d} \bar{f}}{\mathrm{~d} t}+p W \bar{f}=0, \bar{f}(p, 0)=\bar{f}_{0}$

where $p$ is transformation parameter. Then

$\bar{f}(p, t)=f_{0} \mathrm{e}\left[-p \int_{0}^{t} W(t) \mathrm{d} t\right]$.
Going back to the originals we obtain [9]

$f(r, t)=f_{0}\left[r-\int_{0}^{t} W(t) \mathrm{d} t\right]$.

Normal distribution is transformed into

$f(r, t)=\frac{1}{N} \mathrm{e}\left\{-\beta\left[r-\int_{0}^{t} W(t) \mathrm{d} t-\bar{r}\right]^{2}\right\}$,

The parameter of in Eq. 8 is the value of the initial surface energy of a polydisperse mix $\Delta G^{\mathrm{o}}=\sigma \times S_{\text {mol }}$, Joules per $\mathrm{mol}^{\mathrm{e}}$, $\left(S_{\mathrm{mol}}\right.$, square meter per mole; total particle surface per mol of dissolving matter) and is calculated from the condition of dissolution of each particle as follows:

$S_{\mathrm{p}}=4 \pi r^{2}$,

$V_{\mathrm{p}}=\frac{4}{3} \pi r^{3}$, 
surface and size of a particle with radius $r$. The volume of a mole of matter (molar mass $M$, grams per mole; density $\rho$, gram per cubic centimeter) is

$V_{\mathrm{M}}=\frac{M}{\rho}$.

The surface and size of an "average" particle is determined by statistical averaging based on distribution function $f_{0}(r)$

$$
\begin{aligned}
& \bar{S}_{\mathrm{p}}=4 \pi\left\langle r^{2}\right\rangle_{0}=4 \pi \int_{0}^{\infty} r^{2} f_{0}(r) \mathrm{d} r, \\
& \bar{V}_{\mathrm{p}}=\frac{4}{3} \pi\left\langle r^{3}\right\rangle_{0}=\frac{4}{3} \pi \int_{0}^{\infty} r^{3} f_{0}(r) \mathrm{d} r .
\end{aligned}
$$

Consequently, the quantity of particles in a mole of dissolving matter is

$$
\bar{N}_{0}=\frac{V_{\mathrm{M}}}{\bar{V}_{\mathrm{p}}}=\frac{3 V_{\mathrm{M}}}{4 \pi\left\langle r^{3}\right\rangle_{0}} .
$$

Thus, the total surface of particles per mole of matter is

$$
S_{\mathrm{mol}}=\bar{N} \times \bar{S}_{\mathrm{p}}=\frac{3 V_{\mathrm{M}}}{4 \pi\left\langle r^{3}\right\rangle_{0}} \times 4 \pi\left\langle r^{2}\right\rangle_{0}=\frac{3 V_{\mathrm{M}}\left\langle r^{2}\right\rangle_{0}}{\left\langle r^{3}\right\rangle_{0}} .
$$

Finally, the average surface energy of particles is

$$
\overline{\Delta G^{\circ}}=\frac{3 \sigma M\left\langle r^{2}\right\rangle_{0}}{\rho\left\langle r^{3}\right\rangle_{0}} \text {. }
$$

For monodisperse mixtures of particles with radius $r$, the surface energy might equal

$\Delta G_{0}=\frac{3 \sigma M}{\rho r}$.

For distribution, the results obtained from Eqs. 13 and 14 are practically the same if in Eq. 14 the radius of the "homogeneous" mix is taken as $\bar{r}=\langle r\rangle_{0}=\int_{0}^{\infty} \operatorname{rf}_{0}(r) \mathrm{dr}$. Given the small size of particles compared to the electrode's size, the concentration field next to its surface $c(x, t)$ can be estimated by solving the diffusion problem, where the electrode is considered as a continuously operating infinite plane source [11]. In this case, concentration depends only on the $x$-axis perpendicular to its surface and as such is described as follows:

$c(x, t)=\frac{1}{\sqrt{4 \pi D}} \int_{0}^{t} \mathrm{e}\left\{-\frac{(x-\xi)^{2}}{4 D(t-\tau)}\right\} \frac{\phi(\tau) \mathrm{d} \tau}{\sqrt{t-\tau}}$.

where $\phi(\tau)$ is the source output per second as per surface area unit is determined by total current $i$; $\zeta$ is the coordinate of source plane in the taken frame of reference $x$. In our case

$\phi(t)=\frac{i}{\mathrm{nFS}_{e}}$.
This result can be obtained by using the fractional differention method that is done in Electronic supplementary material (ESM), part 1.

By placing the coordinate origin on the electrode surface $(\zeta=0)$, we can calculate the ion concentration on the surface $(x=0)$ via Eq. 15. The advantage of undertaking this approach is that it makes the detailed review of dilution kinetics as well as the consideration of features of particles' mutual arrangement irrelevant. At the same time, it shows the relation between concentration and experimentally measured integral current in a simpler way.

To calculate the relation between the total oxidation current of all particles on the electrode surface and the task parameters (potential sweep rate, $v$; surface free energy of the particles, $\Delta G^{\circ}$ ), it is advisable to use the amount of matter (charge) which has not diluted by the time $t$, based on Eq. 17

$$
\begin{aligned}
& y(t)=\frac{N(t)\left\langle r^{3}\right\rangle}{N_{0}\left\langle r^{3}\right\rangle_{0}}=\int_{0}^{\infty} \frac{r^{3}}{\left\langle r^{3}\right\rangle_{0}} f(r, t) \mathrm{d} r, \\
& \left\langle r^{3}\right\rangle_{0}=\int_{0}^{\infty} r^{3} f_{0}(r) \mathrm{d} r
\end{aligned}
$$

The model is closed with the total charge balance equation (Eq. 18)

$Q=Q_{0}-\int_{0}^{t} i(\tau) \mathrm{d} \tau ; \quad y=\frac{Q}{Q_{0}}=1-\frac{1}{Q_{0}} \int_{0}^{t} i(\tau) \mathrm{d} \tau$.

Differentiating Eq. 18 by $t$ results in the equation with regard to measured current

$\frac{\mathrm{d} y}{\mathrm{~d} t}+\frac{1}{Q_{0}} i(t)=0$,

which, considering Eqs. 12 and 20, enter into

$i(t)=\frac{3 Q_{0} W(t)}{\left\langle r^{3}\right\rangle_{0}} \int_{0}^{\infty} r^{2} f_{0}\left\{r-\int_{0}^{t} W \mathrm{~d} t\right\} \mathrm{d} r ; \quad i(t)=F[i(t), t]$

Another approach is given in ESM, part 2.

Eq. 19 enables to calculate current $i(t)$ dependent on to task parameters.

The initial conditions $(t=0)$ are given as follows:

$$
\begin{aligned}
& i(0)=0 ; \quad c(0)=0 ; \quad E=E_{\text {in }} ; \\
& W(0)=A a_{\mathrm{M}} \mathrm{e}\left[\frac{n \beta \beta}{R T}\left(E_{\text {in }}-E_{0}\right)+\frac{\beta \Delta G^{\circ}}{R T}\right] .
\end{aligned}
$$

Figure 1 shows the voltammograms of electrooxidation of nanoparticle ensembles calculated with the polydisperse (disp) model. Calculations were performed for gold (see parameters in Fig. 1 caption). As is shown in Fig. 1, smaller particle size results in a stronger dispersion effect. Figure 2 demonstrates the relation between 
(a) $E_{\mathrm{m}}$, (b) $I_{\mathrm{m}}$, (c) $I_{\mathrm{m}} / Q_{0}$ and the average quadratic deviation of the initial particle size distribution $(D$, disp). As is easily observed, when dispersion increases, the peak potential shifts to more positive values, $I_{\mathrm{m}}$ and $I_{\mathrm{m}} / Q_{0}$ decrease, and electrooxidation occurs in a wider range of potentials. These features explain the earlier mentioned small differences between the calculated and experimental data observed in $[4-6,8]$.
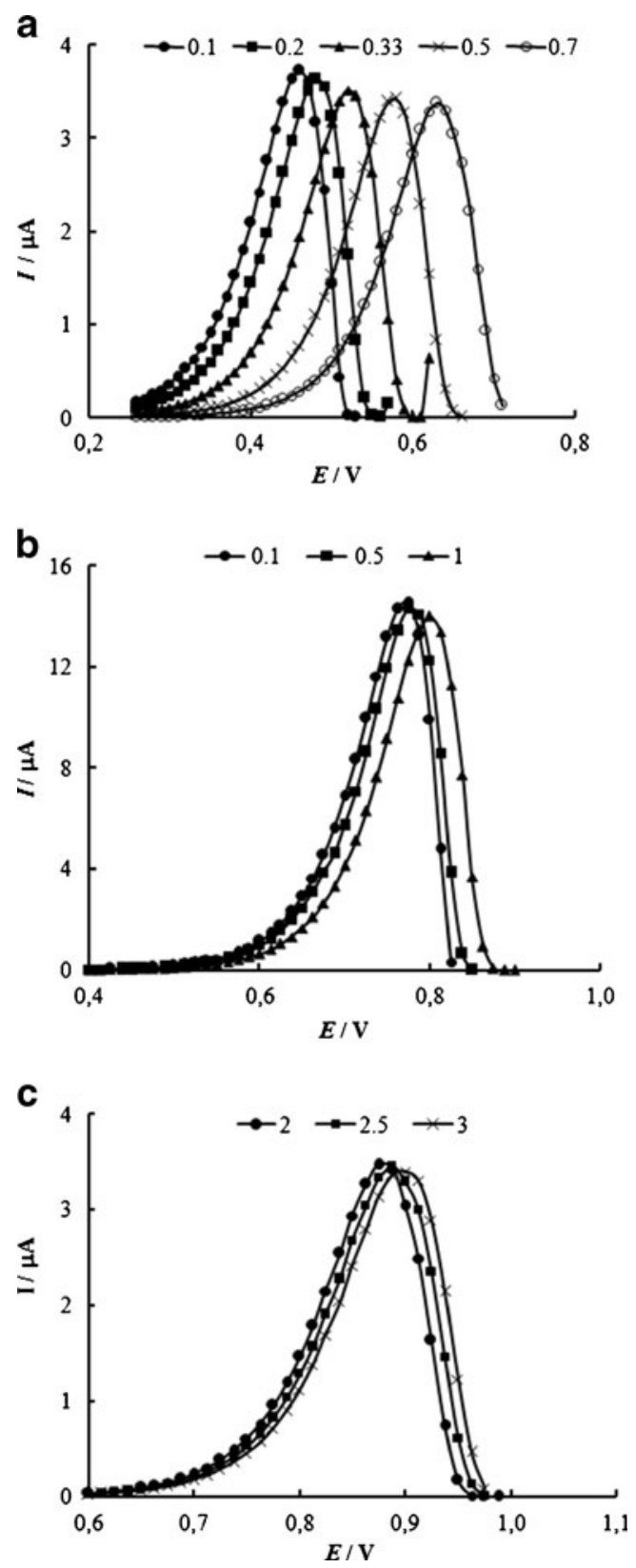

Fig. 1 Electrooxidation volammograms calculated for gold nanoparticles: a $r_{\mathrm{av}}=1 \mathrm{~nm}, \mathbf{b} r_{\mathrm{av}}=3 \mathrm{~nm}, \mathbf{c} r_{\mathrm{av}}=6 \mathrm{~nm}$ and various degrees of dispersion $D$ (given at the top). Calculation parameters: $k_{\mathrm{S}}=3 \times 10^{-8} \mathrm{~cm}$ $\mathrm{s}^{-1}, n=1, E^{0}=0.95 \mathrm{~V}, M=197 \mathrm{~g} \mathrm{~mol}^{-1}, \rho=19.3 \mathrm{gcm}^{3}, \sigma=1,200 \mathrm{din}$ $\mathrm{cm}^{-1} ; \mathbf{a} Q_{0}=8.88 \mu \mathrm{C}, \mathbf{b} Q_{0}=30 \mu \mathrm{C}, \mathbf{c} Q_{0}=8.88 \mu \mathrm{C}$
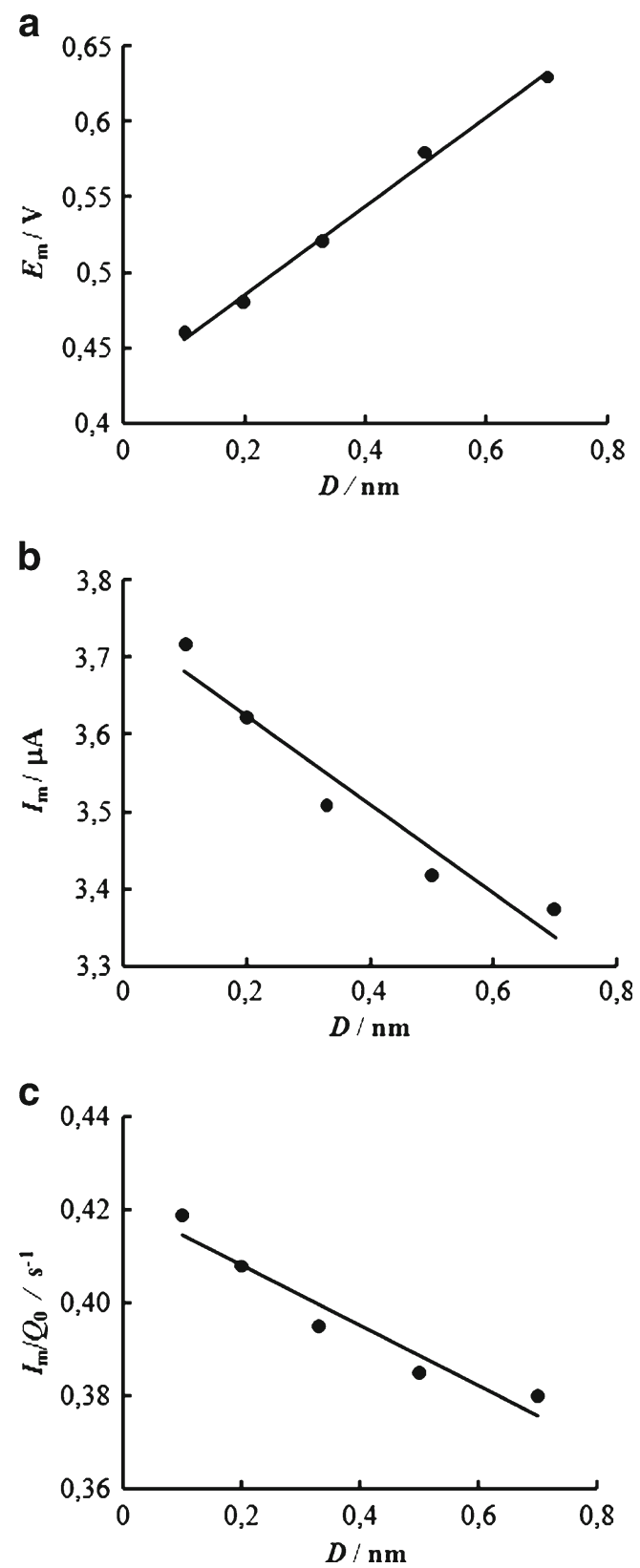

Fig. 2 Relation between a $E_{\mathrm{m}}, \mathbf{b} I_{\mathrm{m}}, \mathbf{c} I_{\mathrm{m}} / Q_{0}$ and $D$, calculated for a mixture of particles $r_{\mathrm{av}}=1 \mathrm{~nm}$

\section{Experimental section}

Reagents, instruments, and procedures

Reagents (high purity and extra high purity grade): acids: $\mathrm{HCl}$ (Reachim, Russia) and $\mathrm{HNO}_{3}$ (Chimreaktivsnab, Russia); sodium citrate (Na-cit) (Labtech, Russia), $\mathrm{HAuCl}_{4}$. All solutions were prepared with deionized water (specific resistance $<18 \mathrm{M} \Omega \mathrm{cm}$, DBC-M/1HA (18), Russia).

Electrochemical measurements were done using a semiautomatic inverse voltammetric analyzer IVA-5 (Russia) and a three-electrode electrochemical cell. A glassy carbon 
rod was used as an auxiliary electrode; a saturated silver/ silver chloride electrode (SCE; $E=0.22 \mathrm{~V}$ vs NHE) was used as a reference electrode. Screen-printed graphite containing electrodes (SPGE; IVA Ltd, Russia) on basis of ink CIRCALOC 6971 (Lord, USA) ex situ modified with gold nanoparticles and gold-sputtered (hereafter "bulk") electrodes were used as working electrodes.

Voltammograms of gold particle electrooxidation were recorded at a linear potential sweep in the range from 0.5 to $1.4 \mathrm{~V}$ at a scan rate $0.05 \mathrm{~V} \mathrm{~s}^{-1}$ using $0.1 \mathrm{M} \mathrm{HCl}$ as a background electrolyte. The gold oxidation potential $\left(E_{\mathrm{m}}\right.$, volts), maximum current $\left(I_{\mathrm{m}}\right.$, micro-ampere), quantity of electricity $\left(Q_{0}\right.$, micro-Coulomb) which corresponded to the quantity of matter participating in the electrochemical process, as well as V-peak half width $\left(\Delta E_{1 / 2}\right)$ were measured. $Q_{0}$ was determined by graphical integration of the area below the voltammograms of gold electrooxidation. All potentials are given relative to SCE.

Synthesis of Au nanoparticles was described in our previous work [4]. Au nanoparticles were synthesized by chemically reducing an aqueous solution of $\mathrm{HAuCl}_{4}$ with sodium citrate. Sols containing gold particles of different sizes were prepared varying the ratio $\mathrm{C}\left(\mathrm{AuCl}_{4}^{-}\right) / \mathrm{C}(\mathrm{Na}$-cit). Three samples of gold sols with varying particle sizes were obtained at $\mathrm{C}$ $\left(\mathrm{AuCl}_{4}{ }^{-}\right) / \mathrm{C}(\mathrm{Na}-\mathrm{cit})=1: 5, \mathrm{C}\left(\mathrm{AuCl}_{4}{ }^{-}\right) / \mathrm{C}(\mathrm{Na}-\mathrm{cit})=1: 2$ and $\mathrm{C}$ $\left(\mathrm{AuCl}_{4}{ }^{-}\right) / \mathrm{C}(\mathrm{Na}-\mathrm{cit})=1: 1$. Synthesized sols were of different colors. Gold sol synthesized at $\mathrm{C}\left(\mathrm{AuCl}_{4}{ }^{-}\right) / \mathrm{C}(\mathrm{Na}-\mathrm{cit})=1: 5 \mathrm{had}$ a deep red color ("red" sol, $\mathrm{Au}_{\mathrm{red}}$ ). Gold sols synthesized at $\mathrm{C}$ $\left(\mathrm{AuCl}_{4}{ }^{-}\right) / \mathrm{C}(\mathrm{Na}-\mathrm{cit})=1: 2$ and at $\mathrm{C}\left(\mathrm{AuCl}_{4}{ }^{-}\right) / \mathrm{C}(\mathrm{Na}-\mathrm{cit})=1: 1$ had violet ("violet" sol, $\mathrm{Au}_{\text {vio }}$ ) and blue ("blue" sol, $\mathrm{Au}_{\text {blue }}$ ) colors in transmitted light, respectively. The color becomes less intensive when the sol concentration decreases. For synthesis of gold nanoparticles, a magnetic stirrer with controlled heating IKA RCT basic (Germany) was used. Gold sols with varying concentrations were prepared by diluting the initial sol containing of $0.1970 \mathrm{gL}^{-1} \mathrm{Au}(\mathrm{III})$ in terms of $\mathrm{Au}(\mathrm{III})$. All synthesized sols were stored at $+4{ }^{\circ} \mathrm{C}$ in the dark.

Immobilization of gold particles on the electrode surface was carried out by placing a drop of $3 \mu \mathrm{L}$ of gold sol, using a single-channel micropipette with variable volume dosage $0.5-10 \mu \mathrm{L}$ (Lenpipet, Russia), on the working area of the electrode and left at ambient conditions in the open air until completely dry. SPGE modified with red, violet, and blue sols are hereafter SPGE/ $/ u_{\text {red }}, \mathrm{SPGE} / \mathrm{Au}_{\text {vio, }}$, and SPGE/ $\mathrm{Au}_{\text {blue, }}$ respectively.

The bulk gold electrode was produced by vacuum metal sputtering from the wire surface $(d=0.2 \mathrm{~mm}, 99.99 \%$ pure gold) onto a polyethyleneterephthalate strip $(0.25 \mathrm{~mm})$ by a VUP-4 instrument [3]. The deposited gold layer was 0.5$1.0 \mu \mathrm{m}$ thick. Cementit 3172 glue was applied to isolate the $0.10 \mathrm{~cm}^{2}$ working area on the electrodes.

A cooling centrifuge $3 K 30$ (Sigma Laborzentrifugen, Germany) was used for centrifugation of gold sols.
Microscopic studies of gold sols immobilized on the SPGE surface were performed with a scanning electron microscope LEO 982 (Carl Zeiss, Germany).

\section{Results and discussion}

We have previously described [3] transmission electron microscopy (TEM) for red and blue gold sols, SEM imaging of the bulk electrode's surface and SPGE modified with $3 \mu \mathrm{L}$ of red and blue gold sol (concentration $0.1970 \mathrm{gL}^{-1}$ $\mathrm{Au}(\mathrm{III})$ in terms of $\mathrm{Au}(\mathrm{III})$ and corresponding bar charts of particle size distribution are described in). According to the results, an average particle radius on the surface of SPGE/ $\mathrm{Au}_{\text {red }}$ is $10 \mathrm{~nm}$, while the dominant average radius on the surface of SPGE/Aublue is $150 \mathrm{~nm}$.

Figure 3 presents photomicrographs of the SPGE surface, modified with $3 \mu \mathrm{L}$ of tenfold diluted red gold sol with a concentration of $0.0197 \mathrm{gL}^{-1}$ in terms of $\mathrm{Au}$ (III; Fig. 3a) and the initial sol with a concentration of $0.1970 \mathrm{gL}^{-1}$ in terms of Au (III; Fig. 3b). On the SPGE surface, gold particles are shown as white spots mainly located on the elevated (gray) areas. In Fig. 3, morphologically the surface of the examined gold electrodes varies significantly. When the sol-modifier concentration increases, an array of nanoparticles on the SPGE surface grows. Distribution of nanoparticles becomes uneven, and the distance between them reduces. High concentrations of red sol nanoparticles lead to the formation of ensembles consisting of two to five gold nanoparticles. Figure 3 also shows the bar chart of particle size distribution on the SPGE surface, modified with diluted (Fig. 3c, e) and concentrated (Fig. 3d, f) red sols. Figure 3c and d display the relation between quantitative proportion (number) of particles ( $N$, percent) and the radius, while Fig. $3 \mathrm{e}$ and $\mathrm{f}$ present the relation between the mass (weight) fraction of particles ( $W$, percent) and the radius. As is seen from comparing the two types of bar charts, smaller particles predominate quantitatively, while larger particles lead in terms of weight. For example, if the concentration of red sol particles on the SPGE surface is low, the $7-9 \mathrm{~nm}$ radius particles dominate quantitatively, while the $10-11 \mathrm{~nm}$ radius particles prevail by mass. Taking into account that the electrochemical response is determined by particle mass, in calculations and discussions we will use the radius relating to a certain mass (weight) fraction (Fig. 3e, f) and use the term "weight particle distribution" ( $W$, percent) referring to the distribution of particles by respective radii. The bar charts demonstrate that a higher concentration of the sol-modifier results in a broader distribution of gold nanoparticles by size, and an average particle radius $\left(r_{\mathrm{av}}\right)$ grows. Thus, on the surface of the SPGE modified with diluted sol, the particle radius varies in a narrower range from 6 to $14 \mathrm{~nm}$, the predominant $r=10 \mathrm{~nm}$. On the surface of the 


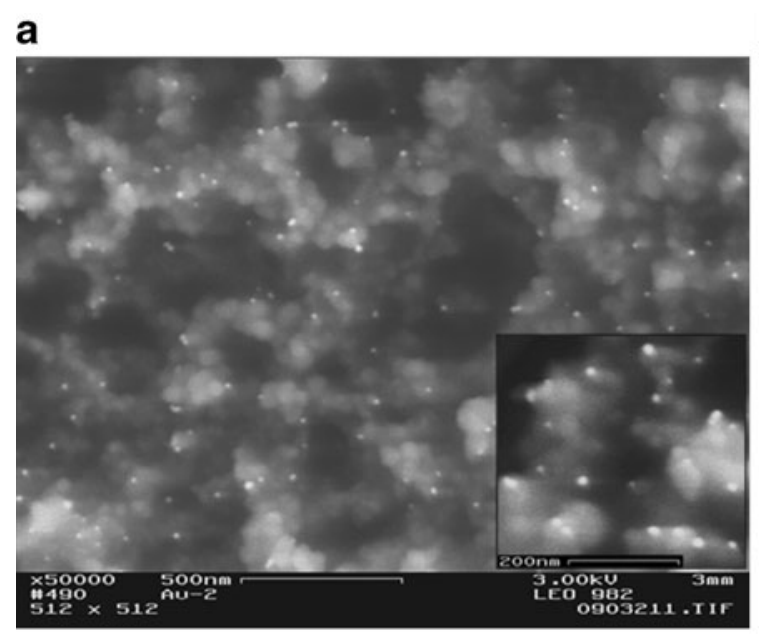

b
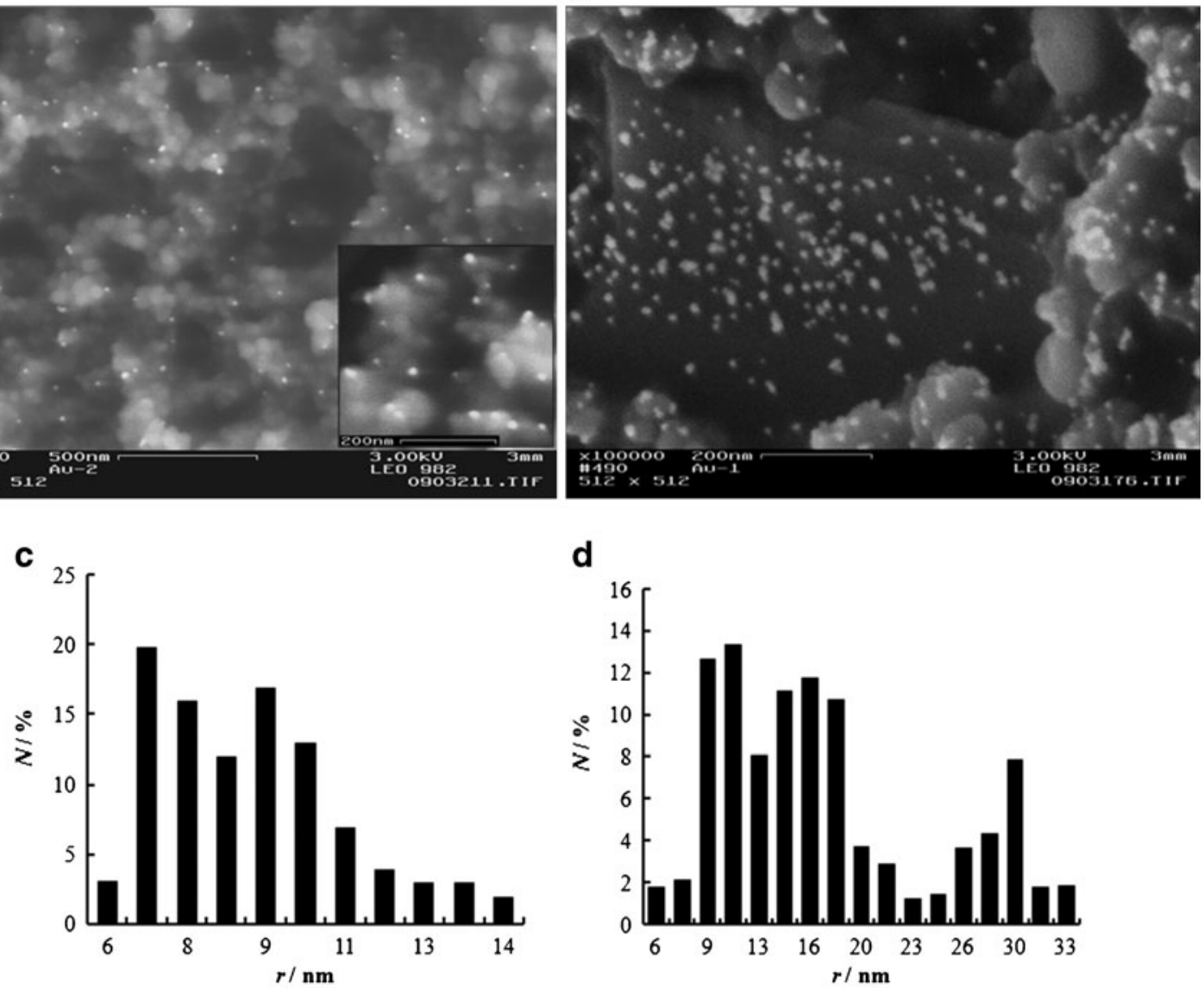

d
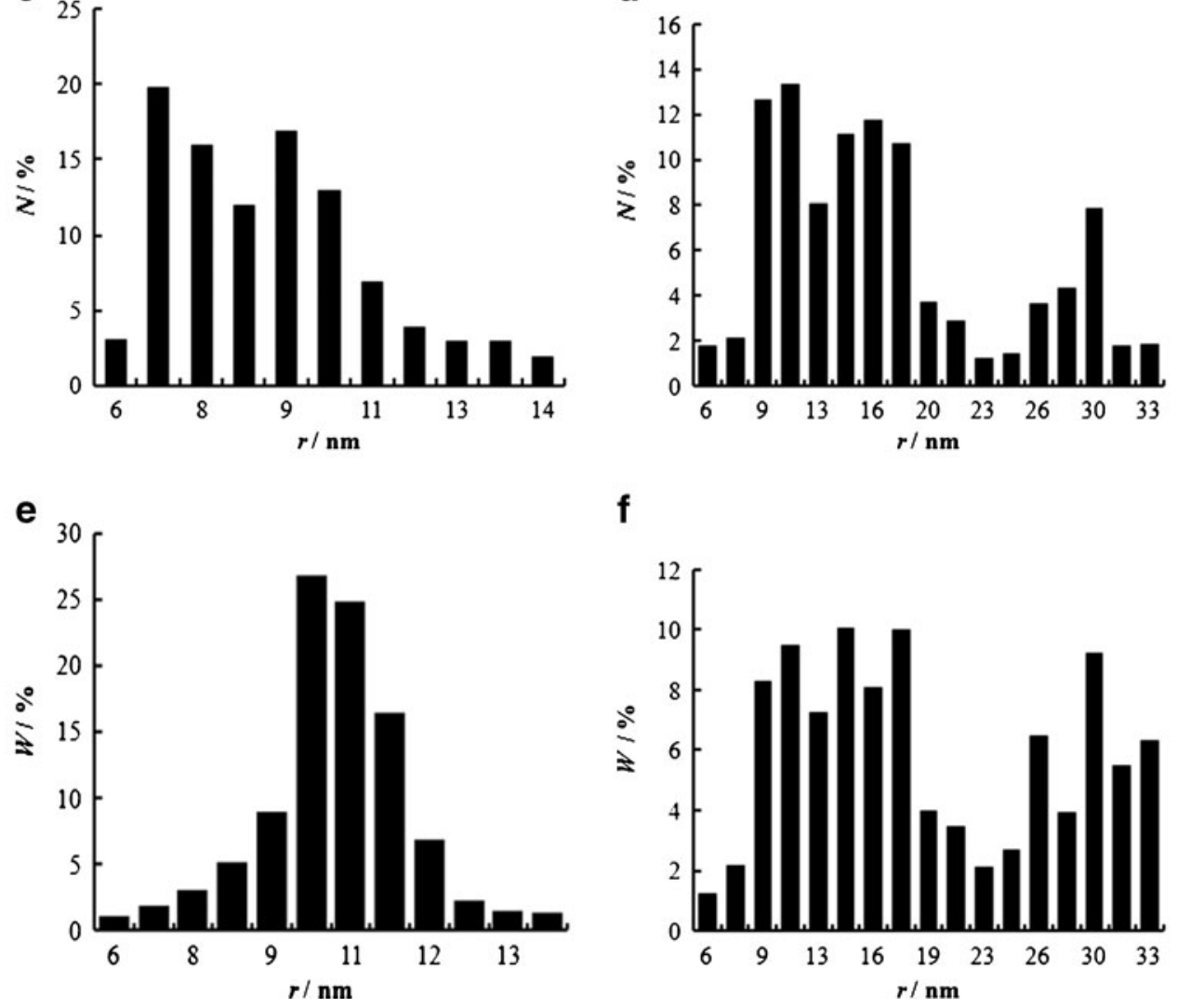

f

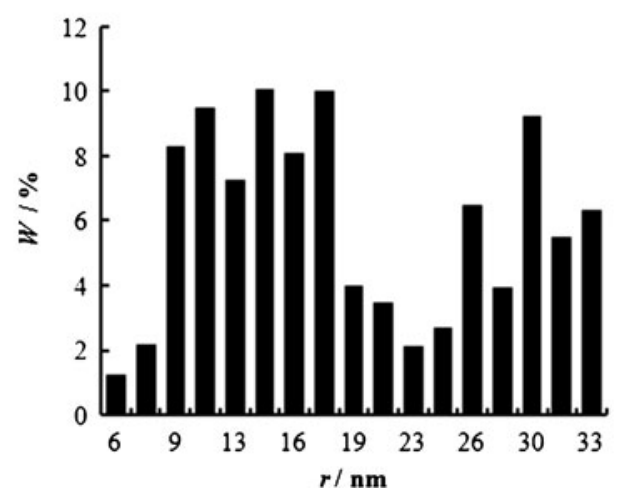

Fig. 3 SEM images of nanoparticles on SPGE surface modified with $3 \mu \mathrm{L}$ of a red gold sol (concentration $0.0197 \mathrm{gL}^{-1}$ ) and $\mathbf{b}$ initial sol (concentration $0.1970 \mathrm{gL}^{-1}$ in terms of $\mathrm{Au}(\mathrm{III})$ ). Bar charts of particle

size distribution on SPGE surface modified with $3 \mu \mathrm{L}$ of red gold sol (concentration, $0.0197 \mathrm{gL}^{-1}(\mathbf{c}, \mathbf{e})$ ) and initial sol (concentration $0.1970 \mathrm{gL}^{-1}$ in terms of $\mathrm{Au}(\mathrm{III})(\mathbf{d}, \mathbf{f})$ )

SPGE modified with highly concentrated red sol, the particle radius varies from 6 to $33 \mathrm{~nm}$ and $r_{\mathrm{av}}=20.9 \mathrm{~nm}$. The appearance on the surface of the electrode modified with a concentrated sol, of additional particles $r<14 \mathrm{~nm}$, results from the formation of aggregates.

Figure 4 presents the experimental voltammograms of particle electrooxidation of red sol diluted 10 times and the

initial sol, background electrolyte is $0.1 \mathrm{M} \mathrm{HCl}$. Table 1 offers the parameters of voltammograms of nanoparticle electrooxidation. The presented data show that higher a concentration of sol, and, consequently, a larger quantity and radius of particles localized on the electrode surface, results in a 20 times increase in the maximum current of particle oxidation. The potential shifts $80 \mathrm{mV}$ to the anodic 
a

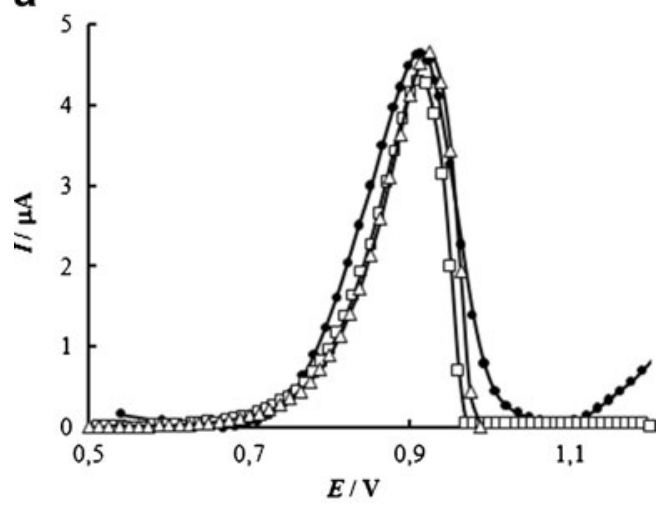

b

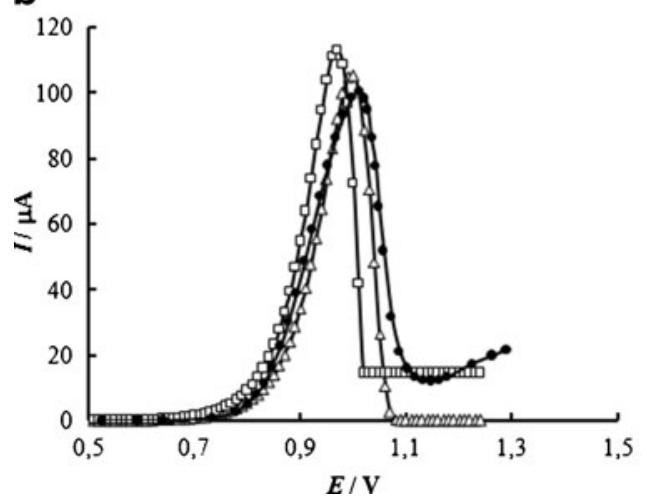

Fig. 4 Experimental (circle) and calculated (with mono (cubic) and disp (triangle) models) voltammograms of electrooxidation of gold particles of red sol from the SPGE surface: a red sol concentration $0.0197 \mathrm{gL}$; b red sol concentration $0.1970 \mathrm{gL}^{-1}$. Experimental conditions: background, $0.1 \mathrm{M} \mathrm{HCl} ; v=0.05 \mathrm{~V} \mathrm{~s}^{-1}$. Calculation parameters as given in Table 1

area; the process of oxidation takes place within a wider range of potentials, as indicated by an increase in $\Delta E_{1 / 2}$ and a decrease in $I_{\mathrm{m}} / Q_{0}$. This is consistent with the abovementioned theoretical considerations.

In our previous paper [4], we presented the experimental and mono model calculated [3] electrooxidation voltammograms of nanoparticles of diluted red sol $\left(r_{\mathrm{av}}=\right.$ $10 \mathrm{~nm}) .3 \mu \mathrm{L}\left(Q_{0}=10.07 \mu \mathrm{C}\right)$ of red sol (concentration $0.0197 \mathrm{gL}^{-1}$ ) was applied to the SPGE surface. The peak of the oxidation of gold nanoparticles, calculated with the mono model, proved to be somewhat narrower than the corresponding experimental peak, and $E_{\mathrm{m}}$ shifted to the cathodic area of potentials. The reason for this apparently lies in the effect of the system's polydispersion on the shape of the current-voltage curve. This is confirmed by comparing (Fig. 4) the experimental voltammograms of gold nanoparticle oxidation and the curves calculated with mono and disp models, as well as parameters given in Table 2. Thus, in the case of electrooxidation of diluted sol nanoparticles, the experimental curves and the curves calculated with the mono and disp models are practically the same, both with regard to $E_{\mathrm{m}}$ and the area of oxidation potentials. Distribution of gold particles by size, in this case, has had no noticeable effect on the shape of the curve, since the physical size of gold particles on the electrode surface changes within a fairly narrow range from 6 to $14 \mathrm{~nm}$. For the initial sol, characterized by a wider distribution of particles by size on the electrode surface (from 6 to $33 \mathrm{~nm}$ ), there is good agreement on the characteristic parameters and shape of the experimental curve and the curve calculated with the disp model. The peak calculated with the mono model is much narrower than the experimental one.

The fact that nanoparticle size distribution on the electrode surface affects the parameters of electrooxidation voltammograms is also confirmed by the results of the study of electrooxidation of nanoparticles deposited on the electrode from supernatant obtained after centrifuging initial gold sols. Since the mass (weight), and consequently, the physical size of the particles depositing during sol centrifugation become smaller with an increase in angular velocity of the centrifuge rotor, supernatant contains even smaller particles. Figure 5 shows the voltammograms of electrooxidation of gold nanoparticles deposited on the electrode from red sol (curve a) and supernatant (curve b). The voltammograms
Table 1 Parameters of experimental and calculated voltammograms of gold nanoparticle electrooxidation for red sol with different concentrations

\begin{tabular}{llcc}
\hline Electrode & & $\mathrm{SPGE} \mathrm{Au}_{\text {red }}\left(0.0197 \mathrm{gL}^{-1}\right)$ & $\mathrm{SPGE} / \mathrm{Au}_{\text {red }}\left(0.1970 \mathrm{gL}^{-1}\right)$ \\
\hline$Q_{0}, \mu \mathrm{C}$ & 12.8 & 276.0 \\
Particle distribution by size, $\mathrm{nm}$ & $6-14$ & $6-33$ \\
$r_{\mathrm{av}}, \mathrm{nm}$ & & 10 & 20.9 \\
$E_{\mathrm{m}}, \mathrm{V}$ & Experiment & 0.927 & 1.008 \\
& Theory mono/disp & $0.900 / 0.919$ & $0.970 / 1.008$ \\
$I_{\mathrm{m}}, \mu \mathrm{A}$ & Experiment & 4.7 & 92.3 \\
& Theory mono/disp & $5.4 / 5.4$ & $113.1 / 105.2$ \\
$\Delta E_{1 / 2}, \mathrm{~V}$ & Experiment & 0.13 & 0.14 \\
& Theory mono/disp & $0.11 / 0.11$ & $0.11 / 0.12$ \\
$I_{\mathrm{m}} / Q_{0}, \mathrm{~s}{ }^{-1}$ & Experiment & 0.37 & 0.33 \\
& Theory mono/disp & $0.42 / 0.42$ & $0.41 / 0.38$ \\
\hline
\end{tabular}


Table 2 Parameters for calculations

\begin{tabular}{|c|c|c|}
\hline Gold sol concentration $\left(\mathrm{gL}^{-1}\right)$ & Mono model calculation [3] & Disp model calculation [This work] \\
\hline \multirow[t]{4}{*}{0.0197} & $r_{\mathrm{av}}=10 \mathrm{~nm}$ & $r_{\mathrm{av}}=10 \mathrm{~nm}$ \\
\hline & & $f(r)=0.38 \times \mathrm{e}\left[-0.45 \times(r-10)^{2}\right]$ \\
\hline & $Q_{0}=12.8 \mu \mathrm{C}$ & \\
\hline & $\Delta G^{\circ}=3,674 \mathrm{Jmol}^{-1}$ & $\Delta G^{\circ}=1,758 \mathrm{Jmol}^{-1}$ \\
\hline \multirow[t]{5}{*}{0.1970} & $r_{\mathrm{av}}=20.9 \mathrm{~nm}$ & $r_{\mathrm{av}}=20.9 \mathrm{~nm}$ \\
\hline & & $f(r)=0.034 \times \mathrm{e}\left[-0.003 \times(r-20.9)^{2}\right.$ \\
\hline & $Q_{0}=275,96 \mu \mathrm{C}$ & \\
\hline & $\Delta G^{\circ}=1,163 \mathrm{Jmol}^{-1}$ & $\Delta G^{\circ}=1,758 \mathrm{Jmol}^{-1}$ \\
\hline & $M=197 \mathrm{~g} \mathrm{~mol}^{-1}, \sigma=1,200 \mathrm{din}$ & $\mathrm{cm}^{-1}, E^{\mathrm{o}}=0.95 \mathrm{~V}, n=1, k_{\mathrm{S}}=3 \times 10^{-8} \mathrm{cms}^{-1}$ \\
\hline
\end{tabular}

were obtained after sol centrifugation $(v=20,000 \mathrm{rpmmin}$, $t=6 \mathrm{~min})$. As before, the oxidation peak is observed at $0.920 \mathrm{~V}$ at the anodic voltammogram of nanoparticles of the initial sol, whereas during electrooxidation of nanoparticles from supernatant the oxidation peak is recorded at $0.864 \mathrm{~V}$. Figure 6 presents a TEM image of gold nanoparticles of red sol and the corresponding bar chart of particle size distribution. The sol contains particles $r=3-5 \mathrm{~nm}$ which could not be registered on the electrode surface due to instrumental limitations of SEM. This sol also contains the predominant quantity of particles $r=6-8 \mathrm{~nm}$. Therefore, it can be concluded that during centrifugation of sol $(v=20,000 \mathrm{rpmmin})$ the $6 \mathrm{~nm}$ radius particles prevail in supernatant, and hence on the electrode surface. In the voltammogram, this is observed as a single peak of oxidation that shifts $60 \mathrm{mV}$ to the cathode area, relative to the peak of oxidation of the initial sol's nanoparticles. Larger particles in these conditions cannot be completely deposited, as indicated by the current recorded at potentials $>0.910 \mathrm{~V}$. Figure 5 shows the calculated voltammograms of electrooxidation of the initial red sol and supernatant nanoparticles, taking into account polydispersion of the samples. In

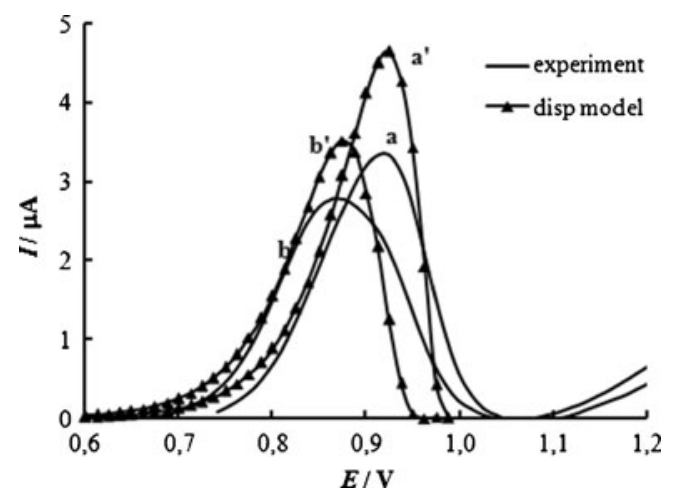

Fig. 5 Experimental and disp model calculated voltammograms of electrooxidation of red sol nanoparticles immobilized on SPGE before $\left(a, a^{\prime}\right)$ and after $\left(b, b^{\prime}\right)$ centrifugation $\left(v=20,000 \mathrm{rpmmin}^{-1}, t=6 \mathrm{~min}\right)$. Experimental conditions: background, $0.1 \mathrm{M} \mathrm{HCl} ; v=0.05 \mathrm{~V} \mathrm{~s}^{-1}$. Calculation parameters: $\left(a^{\prime}\right), r_{\mathrm{av}}=10 \mathrm{~nm}, D=1.06, f(r)=0.38 \times \mathrm{e}[-0.45 \times(r$ $\left.-10)^{2}\right], Q_{0}=9.47 \mu \mathrm{C}, \Delta G^{\circ}=3,595 \mathrm{Jmol}^{-1} ;\left(b^{\prime}\right), r_{\mathrm{av}}=6 \mathrm{~nm}, D=1.76, f$ $(r)=0.23 \times \mathrm{e}\left[-0.16 \times(r-6)^{2}\right], Q_{0}=8.88 \mu \mathrm{C}, \Delta G^{\circ}=5,290 \mathrm{Jmol}^{-1}$. Other parameters: as given in Table 2 calculations of the oxidation curves of particles deposited on the electrode from supernatant, the average particle radius was taken equal to $6 \mathrm{~nm}$ and distribution of particles ranged from 3 to $11 \mathrm{~nm}$, considering the results shown in Fig. 6. When calculating the oxidation peak of the initial gold sol, the above given data on the gold nanoparticles' distribution on the electrode surface (Fig. 3e) were used. In both cases, there is agreement between the experimental and calculated voltammograms for $E_{\mathrm{m}}$. This confirms the assumption that the

a

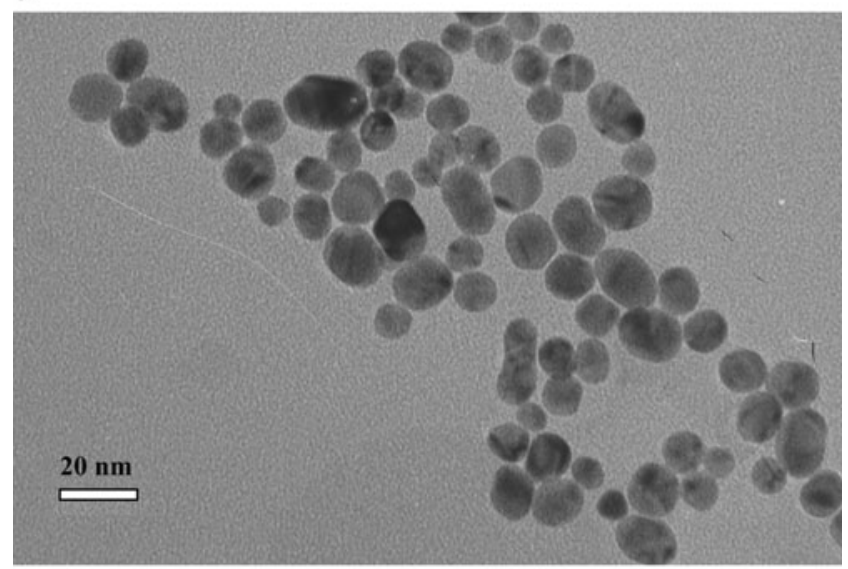

b

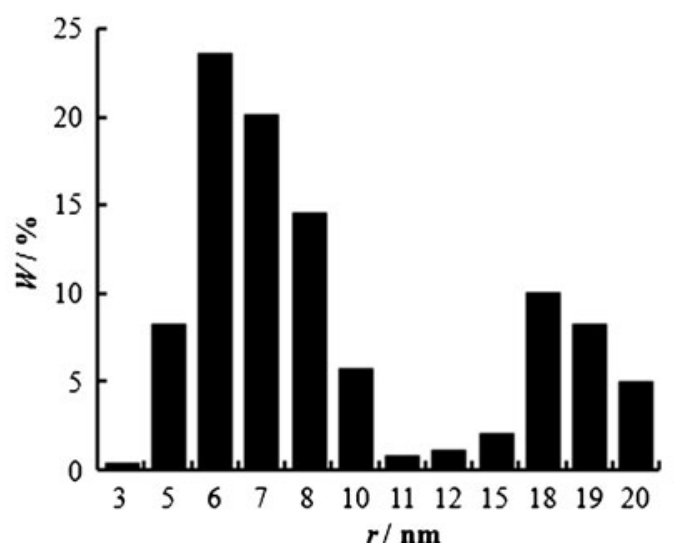

Fig. 6 TEM images of gold nanoparticles in red sol (a) and bar chart of particle distribution by size (b) 
average particle radius on the SPGE surface modified with supernatant is $6 \mathrm{~nm}$. Moreover, the area of oxidation potentials of nanoparticles deposited on the electrode from supernatant is larger than the calculated, which indicates the presence of particles $r<11 \mathrm{~nm}$ on the electrode surface.

To a large extent, the observed patterns seem to be linked to the relation between the potential of the maximum current of electrooxidation and the particles' radius. Figure 7 presents the relation between $E_{\mathrm{m}}$ and $r$ calculated with the mono model [3] (solid curve) and experimental data (dots). As can be observed, when the radius of nanoparticles is smaller, there is a more noticeable difference in their electrochemical activity. For example, if the difference in potentials of electrooxidation $\left(\Delta E_{\mathrm{m}}\right)$ for particles with $r=10$ and $22 \mathrm{~nm}$ is $98 \mathrm{mV}$ (the difference in the radii is only $12 \mathrm{~nm}$ ), then for particles with $r=22$ and $150 \mathrm{~nm} \Delta E_{\mathrm{m}}$ is $59 \mathrm{mV}$ (and the radius difference is $128 \mathrm{~nm}$ ). In this connection during electrooxidation of a set of small-sized nanoparticles, broad oxidation peaks are recorded, even if there is a narrow distribution by particle size. A higher degree of dispersion is demonstrated than during oxidation of larger particles. In this case, the difference in oxidation potentials is insignificant; therefore, fine dispersion leads to narrow peaks in anodic voltammograms.

Similar observations were made in [12]. When ensembles of gold nanoparticles $r \geq 2 \mathrm{~nm}$ were oxidized from the ITO surface immersed into $0.1 \mathrm{M} \mathrm{HClO}_{4}+0.01 \mathrm{M} \mathrm{KCl}$, anodic voltampergrams contains several single peaks, whereas voltammograms of gold nanoparticles $r \geq 2 \mathrm{~nm}$ shows one broad oxidation peak. This pattern can be explained by a large difference in oxidation potentials of gold particles $r=1-$ $2 \mathrm{~nm}$. For example, derived from Plieth equation [13], the difference in oxidation potentials for particles $r=3$ and $4 \mathrm{~nm}$ was $66 \mathrm{mV}$. Moreover, the calculated difference in potentials for particles $r=2$ and $2.4 \mathrm{~nm}$ was also $66 \mathrm{mV}$, and for particles $r=1.4$ and $2 \mathrm{~nm}$ it was $170 \mathrm{mV}$. It is this significant difference in oxidation potentials of very small particles that leads to the presence of separate oxidation peaks in the

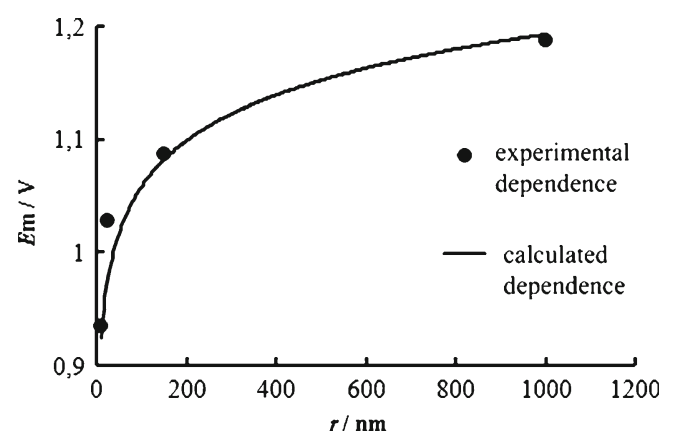

Fig. 7 Mono model [3] calculated and experimental (points; taken from [4]) relations between $E_{\mathrm{m}}$ and $r$. Particle radius: 10, 22, 150 and $1,000 \mathrm{~nm}$. Experimental conditions: background, $0.1 \mathrm{M} \mathrm{HCl} ; v=$ $0.05 \mathrm{~V} \mathrm{~s}^{-1}$. Calculation parameters: $E^{\mathrm{o}}=0.95 \mathrm{~V}, n=1, k_{\mathrm{s}}=3 \times 10^{-8} \mathrm{cms}^{-1}$ voltammograms. The relation between $E_{\mathrm{m}}$ and $r$ stated previously is similar to the relation presented in Fig. 7. Figure 8 shows the voltammogram of oxidation of gold particles $(r=$ 1-50 nm) calculated with the mono model [3]. The experimental conditions were taken from [12]. As is shown in Fig. 8, for radius changes within $1-2.5 \mathrm{~nm}$, in fact, we will observe a noticeable shift of $289 \mathrm{mV}$ to the anodic area, which is described in [12]. With such a strongly pronounced relation between maximum current potential and particle size, we can expect the degree of nanoparticle system dispersion to have a significant effect on the voltammograms' shape. These observations along with those given above are well interpreted within the framework of the proposed approach and provide good evidence of its validity.

An attempt was made to calculate given in [12] curve with three peaks of oxidation of gold particles $r<2 \mathrm{~nm}$. The results of studies of gold sol using TEM presented in this paper demonstrate that the particles' radius changes within the range of $0.6-2 \mathrm{~nm}$. In order to ascertain the size of the particles whose dissolution caused the peaks in Fig. 9, we carried out calculations with the mono model [3] for particles of varying sizes. To construct the total curve, we used the radii corresponding to the calculated curves $(r=0.7,1.5$, and $4 \mathrm{~nm}$. The latter apparently are aggregates formed on the electrode surface), which coincided with the experimental peaks.

Figure 9 presents the calculations made with (a) mono and (b) disp models. Closer correlation to the experiment is observed when dispersion is taken into consideration. The following are the results of experimental studies of electrodissolution of nanoparticle ensembles of red $\left(r_{\mathrm{av}}=10 \mathrm{~nm}\right)$ and violet $\left(r_{\mathrm{av}}=22 \mathrm{~nm}\right)$ gold sols, and their mixtures, which are localized on the electrode surface. This approach enables us to simulate the behavior of both narrowly and widely dispersed nanosystems.

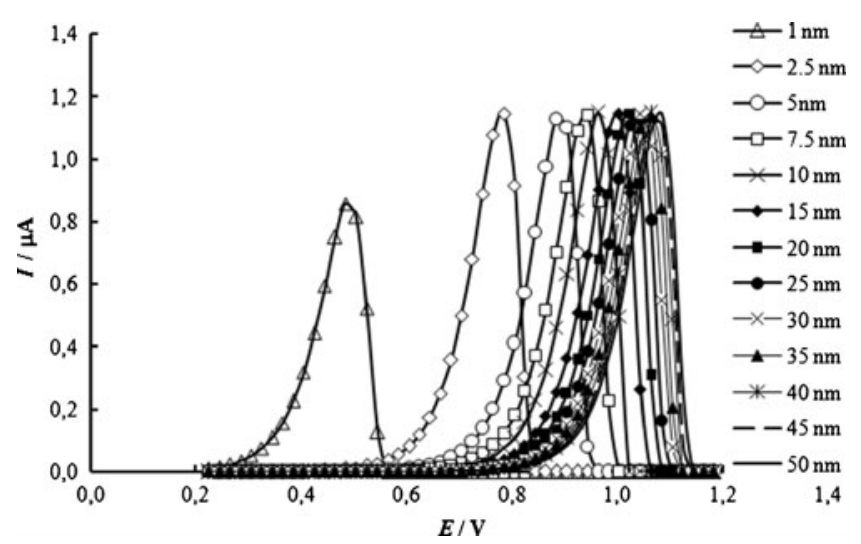

Fig. 8 Calculated voltammograms of gold nanoparticles electrooxidation $(r=1-50 \mathrm{~nm})$. Experimental conditions: $0.1 \mathrm{M} \mathrm{HClO}_{4}+0.01 \mathrm{M}$ $\mathrm{KCl} ; v=0.01 \mathrm{~V} \mathrm{~s}^{-1}$. Calculation parameters: $E^{\mathrm{o}}=1.07 \mathrm{~V}, Q_{0}=10 \mu \mathrm{C}, n=$ $1, k_{\mathrm{S}}=3 \times 10^{-8} \mathrm{cms}^{-1}$ 

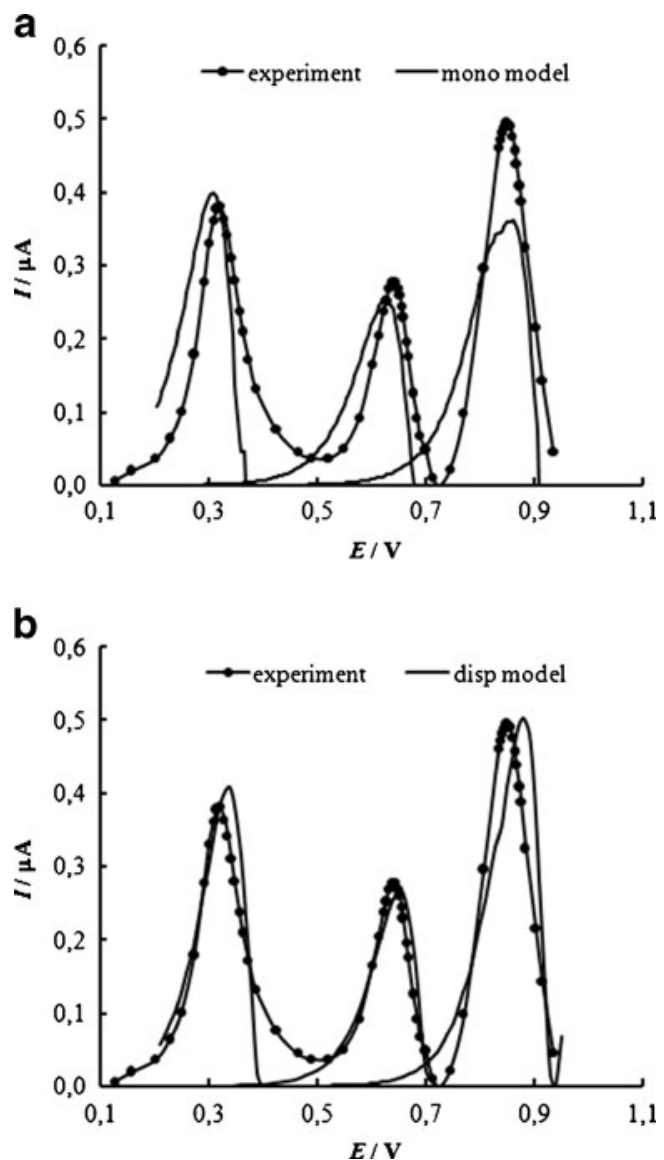

Fig. 9 Experimental (lines) and calculated (points) voltammograms of electrooxidation of gold nanoparticles: a monomodel calculated, $\mathbf{b}$ disp model calculated. Experimental conditions: $0.1 \mathrm{M} \mathrm{HClO}_{4}+0.01 \mathrm{M}$ $\mathrm{KCl}, v=0.01 \mathrm{Vs}^{-1}$. Background is subtracted

Figure 10 presents micrographs of the SPGE surface modified with nanoparticles of violet gold sol $\left(r_{\mathrm{av}}=\right.$ $22 \mathrm{~nm}$ ). Gold nanoparticles on the electrode surface, similar to $\mathrm{SPGE} / \mathrm{Au}_{\mathrm{red}}$, are distributed uniformly; the distance between the particles exceeds their size 3-10 times. On the surface of $\mathrm{SPGE} / \mathrm{Au}_{\mathrm{vio}}$, the radius of the particles varies from 18 to $26 \mathrm{~nm}$, with the predominant radius of $22 \mathrm{~nm}$.

Figure 11 presents the electrooxidation voltammograms of nanoparticles of red $\left(r_{\mathrm{av}}=10 \mathrm{~nm}\right)$ and violet $\left(r_{\mathrm{av}}=22 \mathrm{~nm}\right)$ gold sols and their mixtures. $0.1 \mathrm{M} \mathrm{HCl}$ was used as background electrolyte. Single well-shaped anodic peaks are observed on the voltammograms during electrooxidation of nanoparticles from individual sols. At the same time, when the average size of nanoparticles increases from 10 to $22 \mathrm{~nm}$, the maximum current potential shifts $135 \mathrm{mV}$ to the area with more positive values, which is consistent with prior results [4]. The electrooxidation voltammograms of gold nanoparticles from the SPGE surface, modified with mixtures of red and violet sols, present two anodic peaks. The difference in their half-peak potentials narrows while the volume fraction of red sol nanoparticles $\left(r_{\mathrm{av}}=10 \mathrm{~nm}\right)$ is
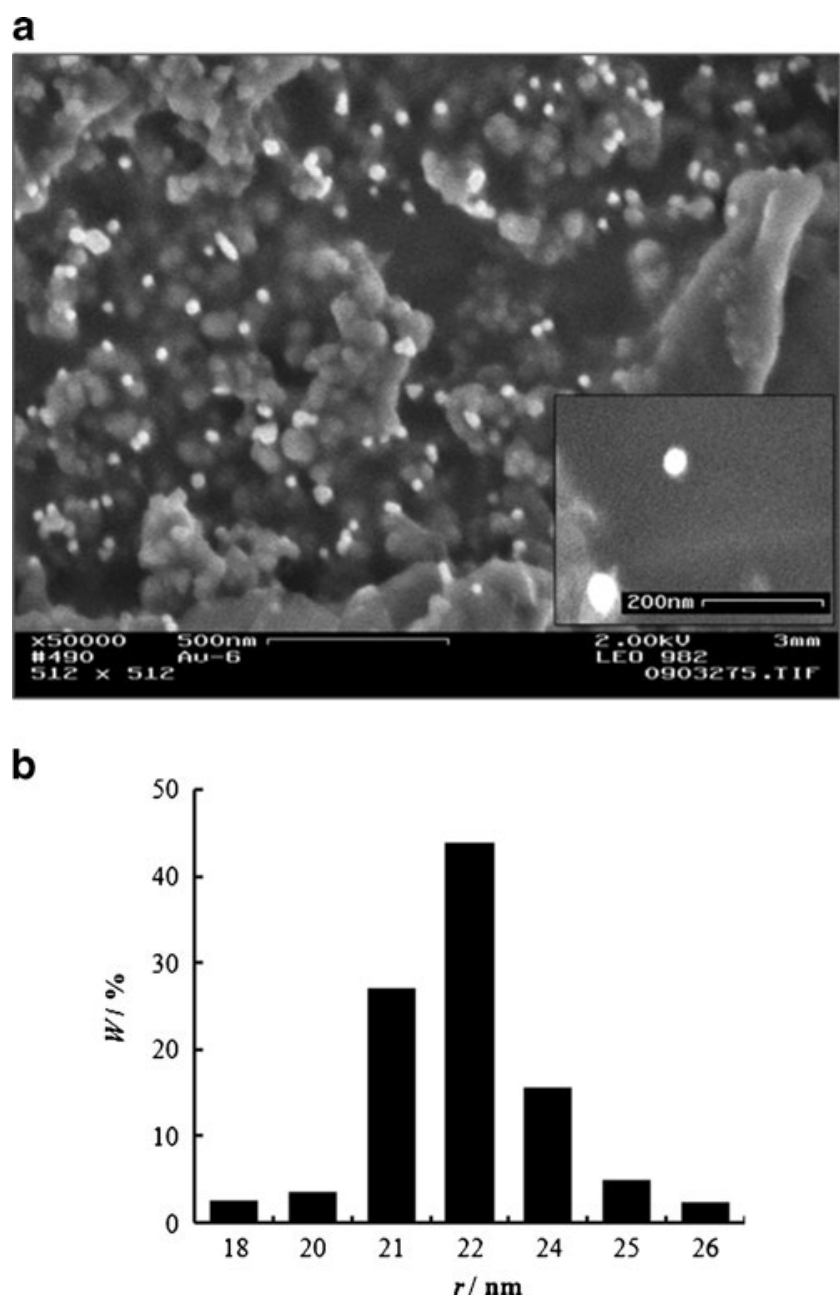

Fig. 10 SEM image of SPGE surface modified with nanoparticles of violet gold sol $\left(3 \mu \mathrm{L}, 0.0197 \mathrm{gL}^{-1} ; \mathbf{a}\right)$ and bar chart of particle size distribution on SPGE surface (b)

increased. With $\left(\mathrm{Au}_{\mathrm{red}}\right) /\left(\mathrm{Au}_{\mathrm{vio}}\right)=4: 1$, the peaks overlap so much that one broad anodic peak is observed.

Regular modification of voltammograms of gold nanoparticle electrooxidation in the transition from individual

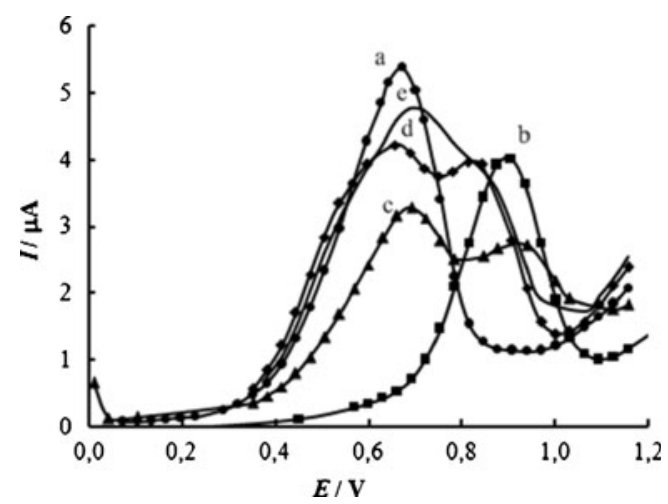

Fig. 11 Anodic voltammograms for nanoparticles of red (a) and violet (b) sols and their mixtures. $\left(\mathrm{Au}_{\mathrm{red}}\right)$ : $\left(\mathrm{Au}_{\mathrm{vio}}\right)$ immobilized on electrode surface: 1:1 (c), 2:1 (d), 4:1 (e). Background: $0.1 \mathrm{M} \mathrm{HCl}, v=0.05 \mathrm{~V} \mathrm{~s}^{-1}$ 
sols to their mixtures on the electrode surface is obviously due to the changing nature of particle distribution by size as well as to a significant increase in the degree of system dispersion. Thus, individual gold sols are characterized by a rather narrow distribution by size (Figs. 4e and 11). The upper range limit for particles of red sol is $14 \mathrm{~nm}$, while the lower limit for particles of violet sol is $18 \mathrm{~nm}$. In this regard, during electrodissolution of gold nanoparticles deposited from $\left(\mathrm{Au}_{\mathrm{red}}\right) /\left(\mathrm{Au}_{\mathrm{vio}}\right)=1: 1$, oxidation of particles of each sol is clearly observed and well separated by potentials. Peak overlapping is not observed. Potentials of anodic current peaks, and consequently, $\Delta E_{\mathrm{m}}$ on voltammograms containing both red $\left(r_{\mathrm{av}}=10 \mathrm{~nm}\right)$ and violet sols $\left(r_{\mathrm{av}}=22 \mathrm{~nm}\right)$ correlate with the values observed in the voltammograms obtained during nanoparticle electrooxidation of individual sols. With an increase in qualitative, and consequently, mass fractions of nanoparticles of red sol in a mixture, anodic current peaks corresponding to electrooxidation of $\mathrm{Au}_{\mathrm{red}}\left(r_{\mathrm{av}}=10 \mathrm{~nm}\right)$ and $\mathrm{Au}_{\mathrm{vio}}\left(r_{\mathrm{av}}=22 \mathrm{~nm}\right)$ gradually converge. At the same time, the dissolution potential of $\mathrm{Au}_{\mathrm{red}}$ shifts to the anodic area. On the contrary, the oxidation potential of $\mathrm{Au}_{\mathrm{vio}}$, shifts to the cathodic area. With $\left(\mathrm{Au}_{\mathrm{red}}\right) /\left(\mathrm{Au}_{\mathrm{vio}}\right)=$ $4: 1$, the voltammogram records a broad anodic peak. It is no longer possible to isolate single processes involving nanoparticles of red or violet sols.

It is natural to conclude that the shape of electrooxidation voltammograms containing a mixture of similar size particles, as well as the anodic current's peak and its position relative to the potential axis, is determined by predominant particle weight. Depending on the degree of gold particles' size distribution on the electrode surface, electrodissolution may occur either in a wider or narrower range of potentials.

\section{Conclusion}

The presented findings show that the degree of dispersion of oxidized particle ensembles localized on the indifferent electrode's surface affects the shape of voltammograms; namely, a broader range of potentials means a higher degree of dispersion and a smaller physical size of particles. Hence, it is right to formulate an inverse problem: to make a judgment about the degree of dispersion of nanoparticle ensembles (in terms of $I_{\mathrm{m}} / Q_{0}$ and $\Delta E_{1 / 2}$ ) and their average size (in terms of the peak potential difference of nanoparticles and bulk metal oxidation) [3].

\section{References}

1. Guntsov AV (2001) Osnovy tyeorii inversionnoĭ vol'tamperometrii. Elektrorastvorenie zarodyshyeǐ osadka. TyumGNGU, Tyumen, p 123

2. Jones SEW, Campbell FW, Baron R, Xiao L, Compton RG (2008) Particle size and surface coverage effects in the stripping voltammetry of silver nanoparticles: theory and experiment. J Phys Chem C 112:17820-17827

3. Brainina KZ, Galperin LG, Galperin AL (2010) Mathematical modeling and numerical simulation of metal nanoparticles electrooxidation. J Solid State Electrochem 14(N6):981-988

4. Brainina KZ, Galperin LG, Vikulova EV, Stozhko NY, Myrzakaev AM, Timoshenkova OR, Kotov YA (2011) Gold nanoparticles electrooxidation: theory and experiment. J Solid State Electrochem 15:1049-1056

5. Brainina KHZ, Galperin LG, Piankova LA, Stozhko NY, Myrzakaev AM, Timoshenkova OR (2011) Bismuth nanoparticles electrooxidation: theory and experiment. J Solid State Electrochem 15:2469-2475

6. Brainina KHZ, Galperin LG, Kiryuhina TY, Galperin AL, Stozhko NY, Myrzakaev AM, Timoshenkova OR (2011) Silver nanoparticles electrooxidation: theory and experiment. J Solid State Electrochem 16:2365-2372

7. Song NW, Park KM, Lee I-H, Huh H (2009) Uncertainty estimation of nanoparticles size distribution from a finite number of data obtained by microscopic analysis. Metrologia 46:480-488

8. Brainina KHZ, Galperin LG, Vikulova EV (2012) Electrochemistry of metal nanoparticles: the effect of substrate. J Solid State Electrochem 16:2357-2363

9. Buevich YA (1966) O kinetike massoobmena polidispersnoĭ sistemy chastits s okruzhayushchyer sredor. Prikl Mekh Tekh Fiz $1: 50-57$

10. Buevich YA, Yasnikov GP (1982) Kinetika rastvoreniya polidispersnoĭ sistemy chastits. Tyeor Osn Khim Tekhnol 16:597-603

11. Karslou G, Yeger D (1964) Teploprovodnost' tverdykh tel. Nauka, Moskva, p 488

12. Masitas AR, Zamborini FP (2012) Oxidation of highly unstable $<4 \mathrm{~nm}$ diameter gold nanoparticles $850 \mathrm{mV}$ negative of the bulk oxidation potential. J Am Chem Soc 134:5014-5017

13. Plieth WJ (1985) The work function of small metal particles and its relation to electrochemical properties. Surf Sci 156:530-535 